\title{
GOTcha: IncRNA-ACOD1 targets metabolism during viral infection
}

Cell Research (2018) 28:137-138. doi:10.1038/cr.2017.153; published online 1 December 2017

\begin{abstract}
Long-noncoding RNAs (IncRNAs) are emerging as important regulators of cellular processes, but few have been functionally characterized in host-pathogen interactions. A recent report in Science demonstrates a mechanistic role for a novel IncRNA in directly binding an essential metabolic enzyme, glutamic-oxaloacetic transaminase (GOT2); this interaction benefits viral replication via alteration of host metabolism.
\end{abstract}

In studying the mammalian transcriptome, it is now apparent that a significant amount of transcribed RNAs are not translated into protein. These noncoding RNAs, formerly considered "junk", actually regulate essential cellular functions. Long-noncoding RNAs (lncRNAs) are a prominent class of RNAs sized 200+ nucleotides that function through varied mechanisms, such as scaffolding protein complexes, sponging microRNAs, and inactivating or stabilizing proteins $[1,2]$. LncRNAs are not yet classified by their function, as their localization and biological mechanisms vary and have not been well elucidated. Nevertheless, it has become clear that lncRNAs regulate vital processes including development, differentiation and metabolism $[1,3]$.

Recent work has demonstrated that specific lncRNAs are important for inflammatory processes. For example, IncRNA-Cox2 was found to be upregulated upon toll-like receptor activation, and this lncRNA interacts with hnRNPs to modify expression of various immune genes [4]. Another so-called lnc-DC is expressed specifically in conventional dendritic cells and directly binds the transcription factor STAT3 to promote differentiation and function of this innate immune cell type [5]. Recently, the field of immunometabolism has highlighted how inflammation and host-pathogen interactions are regulated by metabolic processes, including glycolysis, the TCA cycle, and fatty acid and amino acid metabolism $[6,7]$. While some studies have discovered regulation of metabolism by $\operatorname{lncRNAs}$ and other noncoding RNAs [3, 8, 9], few have linked metabolic reprogramming during infection with IncRNAs. In a new study in Science, Wang et al. [10] investigated the role of IncRNAs during viral infection and found that a specific IncRNA binds and enhances activity of the metabolic enzyme GOT2, which alters host metabolism to benefit viral replication (Figure 1).

To identify lncRNAs involved in viral infection, Wang et al. performed RNA-Seq on macrophages from vesicular stomatitis virus (VSV)-infected mice. LncRNA-ACOD1, named for its proximity to the Acodl ( $\operatorname{Irg} 1)$ gene, stood out as a top hit in this screen, as it was highly upregulated during VSV infection. Importantly, IncRNA-ACOD1 was induced by multiple species of virus but induction was independent of type I interferon. The authors demonstrated that it was a bona fide noncoding RNA using ribosomal profiling and established its expression mainly to innate immune cells during viral infection. Both in vitro siRNA knockdowns and in vivo infection of $\operatorname{lncRNA} A C O D 1^{-/}$ mice show that this IncRNA is required for viral infection, as its loss resulted in decreased viral titers in macrophages and multiple tissues. Furthermore, $\ln$ $c R N A-A C O D 1^{--}$mice were completely protected from mortality during VSV infection compared to wild-type mice, due to low viral load. The authors determined that lncRNA-ACOD1 was essential for viral replication but not for viral entry. Notably, this was independent of Ifnar and Irf3, as well as of host immune responses, evidenced by unchanged cytokine production, autophagy, and apoptosis in macrophages.

One mechanism by which IncRNAs function is to regulate gene expression in cis; however, expression of Acodl and other genes up- and downstream of lncRNA-ACOD1 was not affected by its loss and vice versa. In analyzing the transcriptome and metabolome of infected IncRNA-ACOD $1^{--}$cells, host metabolism was altered compared to wild-type cells. Using RNA immunoprecipitations and mass spectrometry, Wang et al. determined that lncRNA-ACOD1 directly binds the host metabolic enzyme GOT2, an aspartate aminotransferase that converts oxaloacetate and L-glutamate into $\mathrm{L}$-aspartate and $\alpha$-ketoglutarate. Knockdown of GOT2 mimicked the effects of lncRNA-ACOD1 knockdown, whereas addition of exogenous GOT2 rescued loss of IncRNA-ACOD1 and GOT2. Using novel biochemical analyses including eCLIP and mass spectrometry, they demonstrated that a $5^{\prime}$ segment of lncRNAACOD1 directly binds a 15 -amino acid portion of GOT2. Remarkably, the binding of IncRNA-ACOD1 to GOT2 enhanced enzymatic activity of GOT2 


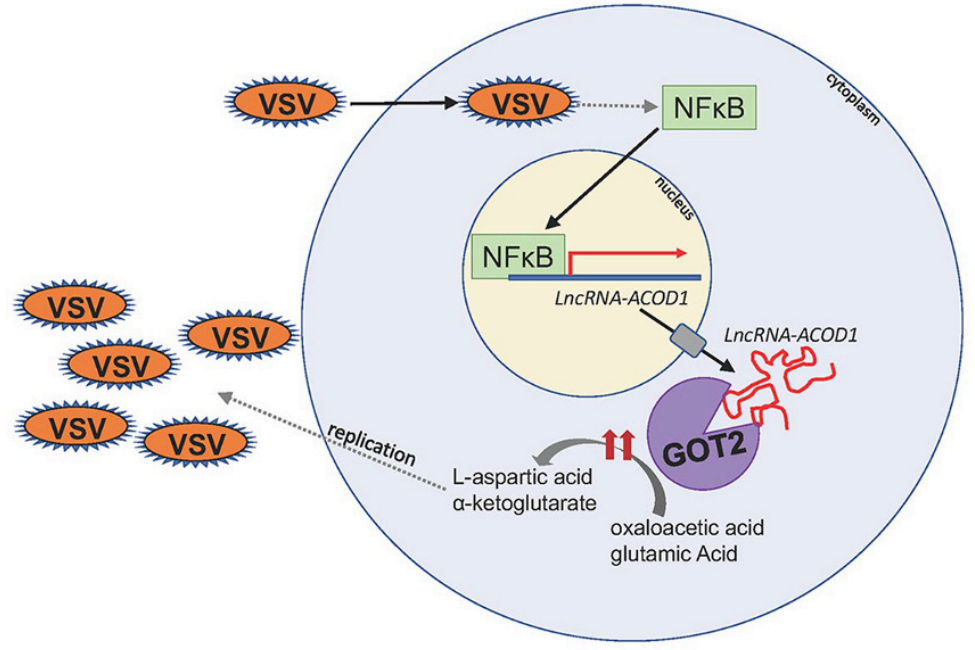

Figure 1 Vesicular stomatitis virus infects a macrophage, leading to activation and nuclear translocation of $\mathrm{NF}_{\kappa} \mathrm{B}$. This allows for transcription of IncRNA-ACOD1, which is then exported into the cytoplasm. LncRNA-ACOD1 directly binds GOT2, boosting its enzymatic activity. GOT2 converts oxaloacetic acid and glutamic acid into $\alpha$-ketoglutarate and L-aspartic acid; these metabolic products promote viral replication.

in vitro. In vivo, mice lacking lncRNAACOD1 had decreased L-aspartate and $\alpha$-ketoglutarate, the metabolic products of GOT2. Finally, exogenous addition of L-aspartate and $\alpha$-ketoglutarate to lncRNA-ACOD $1^{-/}$mice promoted viral infection and increased lethality in these mice. Altogether, this study demonstrates that lncRNA-ACOD1 directly binds and boosts activity of the metabolic enzyme GOT2, thus promoting viral replication.

This paper highlights a novel biochemical role for lncRNAs: directly binding to an enzyme and affecting its activity. LncRNA studies often show regulation at the level of gene expression but here, IncRNA-ACOD1 does not modify chromatin or act in cis. Many lncRNAs function by altering interactions between associated proteins, but in this case, it is noteworthy that IncRNA-ACOD1 directly enhances GOT2 enzymatic activity. While the molecular mechanism behind this is not discussed, one hypothesis is that this lncRNA-ACOD1 makes the active site of GOT2 more accessible to substrate.
Indeed, it is possible that other proteins may be involved in the interaction between lncRNA-ACOD1 and GOT2.

This study also demonstrates a previously unexplored role for GOT2 products L-aspartate and $\alpha$-ketoglutarate in enhancing viral infection. We speculate that these metabolites boost viral infection by increasing nucleotide synthesis and other products needed for replication [11-13], but this needs to be tested. While lncRNA-ACOD1 is induced by $\mathrm{NF}_{\kappa} \mathrm{B}$, its induction and role in bacterial infections and other immune responses have yet to be explored. Because deletion and knockdown of lncRNAACOD1 prevents viral replication without affecting other host responses, this molecule may be a valid therapeutic target to treat viral infections. Nevertheless, it remains to be investigated whether IncRNA-ACOD1 provides any fitness benefit to the host, since it was specifically induced during viral infection to promote viral replication, but did not affect host responses or viability. Further work must be done to clarify the normal function of IncRNA-ACOD1 outside of viral infection, as it may be required for basal host metabolism or in other inducible processes. This paper also raises a more general question on the role that lncRNAs and other noncoding RNAs play in host-pathogen coevolution, as it suggests that lncRNA-ACOD1 provides a fitness benefit to the virus at cost to the host. Overall, Wang et al. used thorough and novel techniques to demonstrate that lncRNA-ACOD1 is a true noncoding RNA that interacts with metabolic enzyme GOT2 to improve its function, opening the door for future studies on lncRNAs in host-pathogen interactions, regulation of immunometabolism, and as targets for infectious disease.

\section{Marah C Runtsch ${ }^{1}$, Luke AJ O’Neill ${ }^{1}$ \\ ${ }^{I}$ School of Biochemistry and Immunology, Trinity Biomedical Sciences Institute, Trinity College Dublin, Dublin 2, Ireland \\ Correspondence: Marah C Runtscha, Luke AJ O'Neill ${ }^{\text {b }}$ \\ ${ }^{a}$ Tel: 353-18962449 \\ E-mail: runtschm@tcd.ie \\ bE-mail: LAONEILL@tcd.ie}

\section{References}

1 Atianand MK, Fitzgerald KA. Trends Mol Med 2014; 20:623-631.

2 Quinn JJ, Chang HY. Nat Rev Genet 2016; 17:47-62.

3 Zhao XY, Lin JD. Trends Biochem Sci 2015; 40:586-596.

4 Carpenter S, Aiello D, Atianand MK, et al. Science 2013; 341:789-792.

5 Wang P, Xue Y, Han Y, et al. Science 2014; 344:310-313

6 O'Neill LA, Kishton RJ, Rathmell J. Nat Rev Immunol 2016; 16:553-565.

7 Olive AJ, Sassetti CM. Nat Rev Microbiol 2016; 14:221-234.

8 Bao MH, Luo HQ, Chen LH, et al. Sci Rep 2016; 6:34161.

9 Yang L, Li P, Yang W, et al. Cell Metab 2016; 24:627-639.

10 Wang P, Xu J, Wang Y, et al. Science 2017; 358:1051-1055.

11 Birsoy K, Wang T, Chen WW, et al. Cell 2015; 162:540-551.

12 Sullivan LB, Gui DY, Hosios AM, et al. Cell 2015; 162:552-563.

13 Sanchez EL, Lagunoff M. Virology 2015; 479-480:609-618. 